\title{
25 Research Square \\ Cost-effectiveness analysis of different methods of treatment of tubal ectopic pregnancy in the south of Iran
}

\section{Bahia Namavar Jahromi}

Shiraz University of Medical Sciences

Elahe esmaili

Shiraz University of Medical Sciences

Mozhgan Fardid

Shahrood University of Medical Sciences

Jafari Abdosaleh

Shiraz University of Medical Sciences

\section{Zahra Kavosi}

Shiraz University of Medical Sciences

\section{Zahra Shiravani}

Shiraz University of Medical Sciences

Khosro Keshavarz ( $\nabla$ khkeshavarz2007@gmail.com )

Shiraz University of Medical Sciences

\section{Research article}

Keywords: ectopic pregnancy, single-dose and double-dose methotrexate, surgery, cost-effectiveness, cost-utility

Posted Date: April 30th, 2020

DOI: https://doi.org/10.21203/rs.3.rs-25488/v1

License: (a) (i) This work is licensed under a Creative Commons Attribution 4.0 International License. Read Full License 


\section{Abstract}

Background: Ectopic pregnancy is one of the most important causes of maternal mortality and infertility that may impose many costs on patients. Today, Surgery and pharmaceutical treatments are the common methods of treating the disease. The aim of this study was to evaluate the cost-effectiveness of different methods of treating tubal ectopic pregnancy in the south of Iran.

Methods: This study was an economic evaluation which analysed and compared the cost-effectiveness and cost-utility of three treatment methods, including single-dose methotrexate, double-dose methotrexate, and surgery in patients with tubal ectopic pregnancy. In this study, a decision tree model was used. The outcomes included in the model were the percentage of successful treatment and the average utility score of each treatment method. The study was conducted from the social perspective and a one-way and probabilistic sensitivity analysis was performed to measure the effects of uncertainty. The analysis of the collected data was performed using Excel and TreeAge software.

Results: The incremental cost-effectiveness ratio of the surgery versus single-dose methotrexate was positive and equal to \$5812 PPP; since it was less than threshold, surgery was considered as a costeffective method. The incremental cost-utility ratio also identified surgery as the best option. Moreover, the results of one-way showed the highest sensitivity to the effectiveness of single-dose methotrexate. Scatter plots also revealed that surgery in $82 \%$ and $96 \%$ of simulations was at the acceptable region compared with single dose and double-dose methotrexate, respectively and below the threshold. It was identified as the more cost effective strategy. Furthermore, the acceptability curves showed that in $81.4 \%$ of simulations, surgery was the most cost effective treatment for thresholds less than 21011 PPP dollars.

Conclusions: Based on the results of the present study, it is recommended that surgery can be used as the first line of treatment for ectopic. Also, the best drug strategy was single dose methotrexate. Since these strategies reduce costs and increase treatment success and QALYs compared to double-dose methotrexate.

\section{Background}

Since the fifth goal of the Millennium Development Goal is to improve the health of mothers, providing care services for vulnerable groups of the community, is of particular importance (1). Ectopic pregnancy is one of the most important causes of mortality and disabilities in women of reproductive age(2). According to the Center for Disease Prevention and Control, this problem occurs in about $2 \%$ of pregnancies, and this small proportion accounts for about $6 \%$ of pregnancy-related deaths(3). The incidence of this disease in Iran is estimated to be $0.26 \%(4)$. The rate of maternal mortality in the country in 2012 and 2013 was $19.9 \%$ and $19.8 \%$, respectively, per thousand live births, and ectopic pregnancy was one of the causes of mortality(1). The incidence of this disease has increased significantly in the last century, but the rate is currently relatively stable. The increase in the past century may be attributed to the rise in utilizing diagnostic technologies such as high sensitivity ultrasounds, increased prevalence of 
sexually transmitted diseases, Assisted Reproductive Techniques (ART), and the use of ovarian stimulants(5). So far, uterine tubes have been the most common site of abnormal implantation that account for $98 \%$ of ectopic pregnancies(2). Ectopic pregnancy may damage the uterine tube or lead to infertility in future(6) and $10-12 \%$ of secondary infertility occurs as a result of such diseases(1). Infertility as a biological, psychological, and social crisis can threaten the health of infertile couples(7). The rapid progress in medical equipment has made it possible to detect ectopic pregnancy via measuring the human chorionic gonadotrophin (HCG- $\beta$ ) subtypes. The measurement of HCG is a precise, non-invasive, and accurate method for diagnosis (2). Methotrexate, as a safe and effective medication, is suggested to be used at different dosages for treating ectopic pregnancy; it is comparable with surgery, rate of success, and rate of pregnancy in future. Laparoscopy or laparotomies are two common methods utilized for the treatment of an ectopic pregnancy. However, the use of these methods depends on the size and location of the ectopic mass and the hemodynamic stability of the patient. It is worth noting that, laparotomy is not always necessary for the treatment of a patient with a ruptured ectopic pregnancy(8). On the other hand, the utilization of a surgical approach depends on two main factors: first, previous experience of a surgeon with laparoscopy, second, the availability of laparoscopic equipment(9). Except for women with hemodynamically unstable condition, laparoscopy is the preferred surgical method for the treatment of ectopic pregnancy. In this procedure, intramuscular methotrexate is administered when there is concern about probability of remnant trophoblastic cells in the tube or in the peritoneal cavity. Usually a singledose of methotrexate is administered for these patients after laparoscopic surgery(6).

It was first used by Tanaka et al. in 1982, and has then been widely accepted as a good method for ectopic pregnancy treatment that is comparable in many respects to surgery (10). Although drug therapy is a good option for many women with ectopic pregnancy without internal bleeding, it is not necessarily the best treatment option for all patients because the result of treatment is not curative in all patients and delays return of the patients to everyday activities(11). Methotrexate is a potent inhibitor of rapidly proliferating cells such as trophoblastic tissue, with an overall resolution rate of approximately 90percent for ectopic tubal pregnancy(6). Today, modern diagnostic methods can help for the early detection of ectopic pregnancy and treatment approaches have become less invasive (12). Because of inappropriate treatment selections, many mothers annually suffer from related complications such as high mortality rate, infertility, and high economic cost (1). Thus, ectopic pregnancy may lead to infertility and lower chances of future fertility for some people, as well as the stress of the recurrence of the disease; accordingly, it can affect the lives of women of reproductive age(10).Successful treatment of ectopic pregnancy can increase the quality of life of patients undergoing treatment, and decrease their suffering from mental illness and physical problems after the disease.

Given the lack of research in this field in the country, the high cost of treating ectopic pregnancy, as well as the risk of infertility or death in the absence of timely and appropriate treatment, it was decided to conduct this research and utilize its results to determine the most cost-effective treatment method for patients with ectopic pregnancy. The results can also help the managers, policymakers, and specialists to make informed decisions about the most appropriate treatment method for the proper use of limited resources. 


\section{Methods}

This economic evaluation project was conducted to retrospectively study the cost-effectiveness of three methods of single-dose methotrexate, double-dose methotrexate, and surgery among 254 women who had non-ruptured tubal ectopic pregnancies from 2015 to 2017 . Out of the 254 women with ectopic pregnancies 116 patients received single-dose methotrexate, 68 cases received double-dose methotrexate, and 70 women were surgically treated. The mentioned patients were selected from the patients who were admitted to two public governmental hospitals in the south of Iran that provide treatment services for such patients. No minors (under 16) were included in our study. The patients were enrolled who had stable hemodynamic conditions and willing to perform the needed follow-ups of medical treatment with methotrexate. The women who were breastfeeding or were Immunocompromised were excluded from the study. Also the cases with alcoholism, active peptic ulcer or hematologic, hepatic, renal or active lung disease were not enrolled in this study. Also, if fetal heartbeats were detected by an ultrasound examination or if the size of the ectopic mass was more than $5 \mathrm{~cm}$ the patients were considered as candidates for the surgical approach and were not enrolled for medical treatment. If a single methotrexate dose was not effective to decrease the BhcG titer to an acceptable value a second dose was prescribed according to the protocol(13). If the size of the mass was larger or if BhcG titer was higher two doses of methotrexate were prescribed since the beginning.

\section{Clinical input}

After enrollment CBC, B-hCG, BUN, Creatinine, Liver Function Tests (LFT) were evaluated for all of the patients and they were recommended to avoid consumption of folic acid, NSAIDs, ASA, exposure to sunlight or sexual intercourse.

For a single Dose method MTX $50 \mathrm{mg} / \mathrm{m} 2$ body surface area (BSA) was given on the first day (day 1 ) and serum B-hCG values were measured on the 4th and 7th day. However, in a double dose protocol MTX $50 \mathrm{mg} / \mathrm{m} 2$ BSA was given on the first and the 4 th day. If B-hCG value declined by $15 \%$ from day 4 to day 7 the treatment was considered to be successful and the Patient was followed by weekly B-hCG titers. If the decline in B-hCG value was less than $15 \%$ at day 7 , the medical therapy was repeated for another cycle. Medical therapy was considered to be failed if the decline in B-hCG was less than $15 \%$ after 3 consecutive cycles(14).

The effectiveness was measured by collecting data on the percentage of the success rate of each method for treating ectopic pregnancy and the utility of each method. The probability of success of each treatment method was measured using a pre-designed form and reviewing the patient's records as well as measuring the utility scores through the EQ-5D inventory and interviews with the patients. The weights for this questionnaire has been estimated by Goudarzi et al. (2016) in Iran(15).

Common complications of treatment using different doses of methotrexate were classified to six different categories: without complications, hematologic complications (including decreased WBC, 
platelet or $\mathrm{Hb}$ ), complications of the digestive system (including oral ulcers, nausea, and diarrhea), renal complications (including, increased BUN and cr, and decreased urine output), liver complications (including rising of liver enzymes or bilirubin), and dermatologic complications (including itching, swelling, or rash). Moreover, the complications of the surgical procedures were classified into five categories: without complications, wound infections, anesthesiology complications, blood transfusions, and other complications. After reviewing the patients' files, and interview, the rate of each type of complication was determined.

\section{Treatment Cost}

Third-person perspective was used in the current study and the data of direct medical costs (DMC) were extracted from medical records of the patients and self-reports. Direct Non-Medical Costs (DNMC) and Indirect Costs (IC) were estimated by using friction cost method and calculated based on the patients' self-reports and data collected by telephone calls. The costs were calculated based on tariffs in 2017 per international dollar (purchasing power parity) with an exchange rate of 12800 Iranian Rials (IRR) per dollar (16).

\section{Model Structure}

Figure 1 shows the decision tree model for single-dose methotrexate, double-dose methotrexate, and surgery. As the diagram shows, using the decision tree model and the TreeAge software, different treatment methods, their related costs, effectiveness, and complications of the disease were analysed. This model was used to assess the success of each treatment method, side effects, and utility in each of the three groups of patients. For each strategy, the success and failure of the method and its side effects were investigated, and the related charts were analysed to select the best treatment strategy.

\section{Cost-effectiveness analysis}

Based on the results of the previous steps, the model was designed in TreeAge software and the extracted data were entered into the model and then the costs, effectiveness, cost-effectiveness and cost-utility analysis for the three methods of treatment were assessed and their ICERs were estimated and compared, using the following formula.

$I C E R=\frac{\operatorname{Cos} t A-\operatorname{Cos} t B}{\text { OutcomeA }- \text { OutcomeB }}$

\section{Sensitivity analysis}


In this study, a one-way and probabilistic sensitivity analysis (PSA) was performed to examine the effects of uncertainty of parameters on the outcomes of the model. In simultaneous multiplex sensitivity analysis, two or more parameters will be changed. However, the more is the number of changing parameters, finding a definite result is difficult. A multiplex sensitivity analysis which is called as scenario analysis assesses the outcomes and effects of different interventions and consequently it estimates the effects of different parameters. There was an attempt to modify the key parameters of the model, including effectiveness and costs for each treatment method. Because of the lack of a specific costeffectiveness threshold in Iran, the threshold in developing countries for each QALYs, as recommended by WHO, was set at one time and three times of gross domestic product (GDP) per capita that was about \$21011 PPP at 2017 for Iran, according to the World Bank report(17).

\section{Results}

In this research, a total of 254 women with tubal ectopic pregnancies were enrolled. Table 1 briefly presents the descriptive data of the patients, including the data on the number of patients, mean age, insurance coverage, number of pregnancies, the mean size of the mass, the mean gestational ages, history of pelvic surgeries, infertility, abortion, and previous ectopic pregnancies.

Table 2 presents direct and indirect costs spent by the three groups for the treatment of tubal ectopic pregnancies. The mean direct medical costs were $\$ 1706$ for the patients receiving single-dose methotrexate, $\$ 2009$ for double-dose methotrexate receivers, and $\$ 2323$ for patients with surgical intervention. Moreover, the mean direct non-medical costs for the three mentioned groups were $\$ 431$, $\$ 684$, and $\$ 229$ and the mean indirect costs for them were $\$ 538, \$ 669$, and $\$ 607$, respectively. Accordingly, direct medical costs accounted for $64 \%, 60 \%$, and $74 \%$ of the total costs; non-medical direct costs accounted for $16 \%, 20 \%$, and $7 \%$ of the total costs; and indirect costs accounted for $20 \%, 20 \%$, and $19 \%$ of the total costs for patients with single-dose methotrexate, double-dose methotrexate and surgical intervention, respectively.

Table 3 presents the results of the cost-effectiveness and Table 4 shows the results of cost-utility analysis. As shown, the expected effectiveness was $0.721,0.87$, and 0.71 ; and the mean costs for each treatment group were 1984, 2850, and 3199 PPP dollars, for treatment via single-dose methotrexate, surgery, and double-dose methotrexate. As shown in Fig. 2-A and Table 3, the results of the costeffectiveness analysis showed that double-dose methotrexate incurred higher costs and had a lower level of effectiveness and was dominated to the two other strategies. Comparison of the two other methods showed that single-dose methotrexate had a mean weighted cost of $\$ 1984$ and a treatment success rate of 0.72 , while surgery had an estimated cost of $\$ 2850$ and a treatment success rate of 0.87 . Thus, the calculated cost-effectiveness ratio was $\$ 5812$, indicating that for each percentage of success in treating ectopic pregnancy by surgery, $\$ 5812$ should be spent. In order to make a decision, we should compare the incremental cost-effectiveness ratio (ICER) with the threshold(18). Therefore, because the ICER is less than the per capita GDP, the surgical treatment method is considered as a more cost-effective option. 
As shown, the expected QALYs of treatment via surgery, single-dose methotrexate, and double-dose methotrexate were $0.81,0.827$, and 0.81 ; and the mean costs for each treatment group were 378,1968 , and 3162 PPP dollars, respectively (see Table 4). Moreover, based on the results of cost-utility analysis, double-dose methotrexate was dominated compared with two other strategies. Comparison of the two methods showed that single-dose methotrexate had a mean weighted cost of $\$ 1968$ and QALYs of 0.827 (lower cost and higher effectiveness), while surgery had an estimated cost of \$378 and QALYs of 0.814 (lower cost and less effectiveness). Thus, the calculated incremental cost-utility ratio was $\$ 92191$, indicating that per additional QALY gained in treating ectopic pregnancy by single-dose methotrexate, $\$ 92191$ should be spent. Therefore, because the ICER is greater than the per capita GDP, the surgical treatment method is considered as a more cost-effective option.

\section{Uncertainty analysis}

As mentioned earlier, the effects of uncertainty were studied using one-way sensitivity analysis and the value of each variable changed by $20 \%$ and the tornado diagram was plotted. The incremental costeffective ratio, as shown in Fig. 3 , had the highest level of sensitivity to the effectiveness of single-dose methotrexate, and the lowest level of sensitivity to the cost of single-dose methotrexate.

Besides, in the current study a second-order Monte Carlo simulation was conducted using 5000 trials for probabilistic sensitivity analysis. The findings of the uncertainty measurement using cost-effectiveness acceptability curves and incremental cost-effectiveness scatter plot (ICER) distribution curves are presented in the following:

Besides, the cost-effectiveness scatter plots revealed that overall surgery compared with single dose and double-dose methotrexate in $82 \%$ and $96 \%$ of simulations was at the acceptable region, below the threshold and identified as the more cost effective strategy (Fig. <link rid="fig4" >4</link>-A and 4-B). The results of the acceptability curves also showed that in $81.4 \%$ of simulations, surgery was the most cost effective treatment for thresholds less than 21011 PPP dollars. The next best drug strategy was single dose methotrexate which was the optimal strategy in $18.48 \%$ of the simulations (Fig. 5).

\section{Discussion}

Ectopic pregnancies must be treated well to terminate pregnancy and to prevent future mortality and infertility(19). Currently, common treatments for this disease are medical therapy using methotrexate with different doses and surgery to remove the mass(20). This study was conducted to determine and compare the cost-effectiveness and cost-utility of the three aforementioned methods for the treatment of ectopic pregnancies to propose the best options for the physicians and policymakers to choose the most cost-effective method for terminating pregnancy and reducing damage to the uterine tubes. Based on the findings of the cost components (Table 2), the mean costs of treatment via single-dose methotrexate, double-dose methotrexate, and surgery were $\$ 2675$, $\$ 3362$, and $\$ 3159$ per single round of treatment. Therefore, the mean cost of treatment per patient treated with single-dose methotrexate was lower than the mean cost of treatment per patient treated by the other two therapies. It seems that the main reasons 
for the differences in the costs are the higher success rate, lack of the need for secondary treatments, fewer side effects, and lower costs. Given that double-dose methotrexate is prone to higher rates of failure, the direct and indirect medical costs are increased and the patient has to spend more time to treat the illness and recover, which in turn increases the indirect $\operatorname{costs}(21,22)$. Therefore, the results of this study are consistent with those of the Creinin et al.'s study that compared the costs of treatment via single-dose methotrexate and surgery(23), and also Sowter et al.'s study that compared direct and indirect costs of single-dose methotrexate and laparoscopic surgery(24). Therefore, as the results showed, direct medical costs, direct non-medical costs, and indirect costs, respectively, accounted for $64 \%, 16 \%$, and $20 \%$ of the total costs spent by the patients receiving single-dose methotrexate, $60 \%, 20 \%$, and $20 \%$ of the total costs spent by the patients receiving double-dose methotrexate, and $74 \%, 7 \%$, and $19 \%$ of the total costs spent by the patients undergoing surgery. Thus, direct medical costs account for the highest cost in all the three treatment methods. In patients receiving single-dose and double-dose methotrexate, the costs of hospital stay accounted for the highest share of direct medical costs, while for the patients who had surgeries the operation costs accounted for the highest share of direct medical costs. These findings are in line with the results of the published study performed by Foulk et al. (25). Furthermore, it seems that the higher costs of surgery is because of the high operation expenses, services, and drugs that the patients take after discharge from the hospital(26). As calculated, the direct medical costs accounted for a higher percentage of the costs(27), and although about $99 \%$ of the patients undergoing surgical treatment were covered by insurance companies, they incurred high direct medical costs that may be attributed to the high costs of operation(28). Therefore, the costs associated with ectopic pregnancy are relatively high.

The results showed that the rate of treatment success was $721 \%$ for single-dose methotrexate, $71 \%$ for double-dose methotrexate, and $87 \%$ for surgery; the findings of this study are in line with the results of Hajenius et al.'s study that compared the effectiveness of laparoscopy, as a surgical procedure, with methotrexate therapy(29), Chaychian's study that compared single-dose and multi-dose methotrexate(30), and Mergenthal et al.'s study that compared the effectiveness of single dose and double-dose methotrexate(31). However, our results are not consistent with those of Al Yasin et al.'s study that compared single-dose and double-dose methotrexate; they found that double-dose methotrexate had a higher success rate than single-dose methotrexate(32). These different results may be induced by the different methods of patient selection. In our study if the BhcG titers were higher, or size of the ectopic mass was larger or the first methotrexate dose was not effective a second dose were give. So, it is logical that these patients would have lower chances to be cured by the medical therapy.

Moreover, results of our study showed that the mean cost of surgery and single-dose methotrexate was $\$ 2850$ and $\$ 1984$, and their treatment success rates were $87 \%$ and $721 \%$, respectively. In other words, surgery was more costly and more cost-effective, but since the ICER was below the threshold, this method was identified as a cost-effective option. This finding is not in line with the results of the studies conducted by Morlock, Alexander, and Yao; they compared methotrexate therapy with laparoscopy and concluded that methotrexate was more-cost effective than surgery $(25,33,34)$ However, Mol et al. 
compared methotrexate and laparoscopic surgery and found that a particular type of surgery was more cost-effective than methotrexate $(35,36)$.

According to the data obtained from the three groups of patients using the EQ-5D questionnaire, the results showed that the patients who received single-dose methotrexate had higher scores in their utility because they underwent less invasive procedures and needed a shorter period of rest after discharge from the hospital. Results of the present study showed that the highest utility scores were observed in single-dose methotrexate group (0.827), double-dose methotrexate group (0.81), and surgery group (0.81), respectively. Moreover, the results of ICER analysis of utility in all the three groups of patients showed that, among the treatment strategies used in the county, single-dose methotrexate had the higher level of cost and QALYs than the surgery method; but the ICER is greater than the per capita GDP and thus, the surgical treatment method is considered as a more cost-effective option. So far, to our knowledge no study has investigated the utility outcome.

The results of sensitivity analysis showed that ICER is not sensitive to the most of parameters which confirms the robustness of the results of the study. As the results of one-way sensitivity analysis revealed that ICER is more sensitive to single-dose methotrexate but low sensitive to other parameters. Although the single-dose effectiveness parameter had the greatest impact on ICER value by $20 \%$ increase on ICER as it increased the baseline ICER from 5812 to approximately \$14000 PPP, however, the new ICER value is still below the threshold level and as a result the surgery is the dominant option.

Besides, scatter plots also demonstrated that surgery in $82 \%$ and $96 \%$ of simulations was at the acceptable region compared with single dose and double-dose methotrexate, respectively and below the threshold. It was identified as the more cost effective strategy. Furthermore, the acceptability curves showed that in $81.4 \%$ of simulations, surgery was the most cost effective treatment for thresholds less than 21011 PPP dollars

In the present study, we directly estimated direct medical costs, direct non-medical costs, indirect costs and effectiveness using patient-level data instead of using data from the published literature. This approach is also seen in Bastani and Kiadaliri in the methodology of their study in two groups of breast cancer patients using adjuvant therapy(36).

This study has also some limitations. As presented in the data collected on demographic characteristics of patients and their background diseases, none of the files reported a history of pelvic infection; this indicates that women are not paying enough attention to such issues or deny and feel embarrassed about it. Women with ectopic pregnancy are worried about the complications such as repeated ectopic pregnancy and subsequent infertility(37). In addition, the number of patients treated with double-dose methotrexate was lower than those treated via the other two methods.

Concerning the generalizability of the results, since methotrexate and surgery in Iran are used for the treatment of ectopic pregnancies and their prices are the same throughout the country, the results of this study can be generalized to other provinces and the whole country. However, in order to generalize the 
results of this study to other countries, it is necessary to address different issues such as epidemiology of the disease, demographic structure, availability of resources, prices, evaluation of outcomes by individuals, thresholds, and the use of various indicators of effectiveness in different studies that may affect the results of the study. Therefore, one must be cautious in generalizing the results to other countries.

\section{Conclusions}

Overall, based on the results of this study and considering the incremental cost-effectiveness and costutility ratio, surgery is a superior treatment strategy and can be used as a high priority method, as compared with single-dose and double-dose methotrexate. Also, the best drug strategy was single dose methotrexate. Furthermore, since their use as the first line of treatment reduces the time duration and helps to manage the costs, as compared with double-dose methotrexate, it is suggested that surgery and/or single-dose methotrexate can be used as the first line of treatment for ectopic pregnancy to reduce the burden of disease and financial burden in the community.

\section{Abbreviations}

PPP

Purchasing Power Parity

QALY

Quality Adjusted Life Years

ART

Assisted Reproductive Techniques

LFT

Liver Function Tests

EQ-5D

EuroQol-5Dimension

DMC

Direct medical costs

DNMC

Direct non-medical costs

IC

Indirect Costs

IRR

Iranian Rials

GDP

gross domestic product

ICER

incremental cost-effectiveness ratio 


\section{Declarations}

- Ethics approval and consent to participate

This Manuscript is retrieved from an MSc thesis supported and approved by Shiraz University of Medical Sciences with the ID number of 95-01-07-13208. The informed consent was verbal because in this study we used telephone contact. Also, the research proposal was approved by ethics committee of Shiraz University of Medical Sciences.

Not applicable

- Consent for publication

Not applicable

- Availability of data and materials

Datasets analysed during the current study are available from the corresponding author on reasonable request.

- Competing interests

The authors declare that they have no competing interests.

- Funding

Not applicable.

- Authors' contributions

BNJ: participate in design of study and supervised whole study and revised the paper critically for important intellectual content. EE: Study concept and design, participated in literature bibliography, Acquisition of data, Analysis and interpretation of data, Drafting of the manuscript, Critical revision of the manuscript for important intellectual content. MF: Drafting of the manuscript and revising the paper AJ: Analysis and interpretation of data and revised the paper critically for important intellectual content. ZK: Drafting of the manuscript, revised the paper critically for important intellectual content. ZSH: participate in design of study and drafting of the manuscript. KHK: Participate in design of study and drafting of the manuscript, and final revision of the manuscript. All authors read and approved the final manuscript.

- Acknowledgements

The authors would like to thank Shiraz University of Medical Sciences, Shiraz, Iran and also Center for Development of Clinical Research of Nemazee Hospital and Dr. Nasrin Shokrpour for editorial assistance.

\section{References}


1. Hasani M, Keramat A, Khosravi A, Oshrieh Z, Hasani M. Prevalence of ectopic pregnancy in Iran: a systematic review and meta-analysis. The Iranian Journal of Obstetrics, Gynecology and Infertility. 2016;19(23):15-23.

2. Speroff L, Fritz MA. Clinical gynecologic endocrinology and infertility: lippincott Williams \& wilkins; 2005.

3. Control CfD, Prevention. Ectopic pregnancy-United States, 1990-1992. MMWR Morbidity and mortality weekly report. 1995;44(3):46.

4. Novak E. Berek \& Novak's gynecology: Lippincott Williams \& Wilkins; 2007.

5. Burkman RT. Berek \& Novak's gynecology. JAMA. 2012;308(5):516-7.

6. Burkman RT. Berek and Novak's gynecology. JAMA. 2007;297(14):1601-4.

7. Pahlavani H, Malakooti K, Shahrokh E. Study of stressors, coping strategies and its relation to mental health in infertile persons. Andeesheh va Raftar. 2002;7(4):79-81.

8. Murphy AA, Nager CW, Wujek JJ, Kettel LM, Torp VA, Chin HG. Operative laparoscopy versus laparotomy for the management of ectopic pregnancy: a prospective trial. Fertility and sterility. 1992;57(6):1180-5.

9. Cunningham FG. Hypertensive disorders in pregnancy. Williams obstetrics. 2005.

10. Tanaka T, Hayashi H, Kutsuzawa T, Fujimoto S, Ichinoe K. Treatment of interstitial ectopic pregnancy with methotrexate: report of a successful case. Fertility and Sterility. 1982;37(6):851-2.

11. Robin F, Lecuru F, Bernard J-P, Mac-Cordick C, Boucaya V, Tourelle R. Methotrexate provides significant cost savings for the treatment of unruptured ectopic pregnancy. Clinical drug investigation. 1998;15(5):405-11.

12. Murray H, Baakdah H, Bardell T, Tulandi T. Diagnosis and treatment of ectopic pregnancy. Cmaj. 2005;173(8):905-12.

13. Berek JS. Novak's gynecology. The Journal of Midwifery \& Women's Health. 2003;48(3):237-8.

14. Hoffman B, Schorge K, Bradshaw L, Halvorson J. Schaffer, \& MM Corton, Williams Gynecology. McGraw Hill Professional; 2016.

15. Goudarzi R, Zeraati H, Sari AA, Rashidian A, Mohammad K. Population-based preference weights for the EQ-5D health states using the visual analogue scale (VAS) in Iran. Iranian Red Crescent Medical Journal. 2016;18(2).

16. Bank W. World Development Indicators [Available from: https://databank.worldbank.org/home.aspx.

17. Bank W. World Development Indicators [Available from: http://databank.worldbank.org/data/reports.aspx?source=2\&series=NY.GDP.PCAP.PP.CD\&country=.

18. Marseille E, Larson B, Kazi DS, Kahn JG, Rosen S. Thresholds for the cost-effectiveness of interventions: alternative approaches. Bulletin of the World Health Organization. 2014;93:118-24.

19. Kadar N, Caldwell BV, Romero R. A method of screening for ectopic pregnancy and its indications. Obstetrics and gynecology. 1981;58(2):162-6. 
20. Fylstra DL. Tubal pregnancy: a review of current diagnosis and treatment. Obstetrical \& gynecological survey. 1998;53(5):320-8.

21. Barnhart KT, Gosman G, Ashby R, Sammel M. The medical management of ectopic pregnancy: a meta-analysis comparing "single dose" and "multidose" regimens. Obstetrics \& Gynecology. 2003;101(4):778-84.

22. Washington $A E$, Katz P. Ectopic pregnancy in the United States: economic consequences and payment source trends. Obstetrics and gynecology. 1993;81(2):287-92.

23. Creinin MD, Washington AE. Cost of ectopic pregnancy management: surgery versus methotrexate. Fertility and sterility. 1993;60(6):963-9.

24. Sowter MC, Farquhar CM, Gudex G. An economic evaluation of single dose systemic methotrexate and laparoscopic surgery for the treatment of unruptured ectopic pregnancy. BJOG: An International Journal of Obstetrics \& Gynaecology. 2001;108(2):204-11.

25. Foulk RA, Steiger RM. Operative management of ectopic pregnancy: a cost analysis. American journal of obstetrics and gynecology. 1996;175(1):90-6.

26. Yao M, Tulandi T. Current status of surgical and nonsurgical management of ectopic pregnancy. Fertility and sterility. 1997;67(3):421-33.

27. Alexander JM, Rouse DJ, Varner E, Austin Jr JM. Treatment of the small unruptured ectopic pregnancy: a cost analysis of methotrexate versus laparoscopy. Obstetrics \& Gynecology. 1996;88(1):123-7.

28. Rulin MC. Is salpingostomy the surgical treatment of choice for unruptured tubal pregnancy? Obstetrics \& Gynecology. 1995;86(6):1010-3.

29. Hajenius P, Engelsbel S, Mol B, Van der Veen F, Ankum W, Bossuyt P, et al. Randomised Trial of Systemic Methotrexate Versus Laparoscopic Salpingostomy in Tubal Pregnancy. Obstetrical \& gynecological survey. 1998;53(1):27-8.

30. Chaichian S, Mehdizadeh A, Akbarian AR. Investigation of Benefits and Risks of Laparascopic Surgery in the Treatment of Ectopic Pregnancy. Razi Journal of Medical Sciences. 2006;12(49):2732.

31. Mergenthal MC, Senapati S, Zee J, Allen-Taylor L, Whittaker PG, Takacs P, et al. Medical management of ectopic pregnancy with single-dose and 2-dose methotrexate protocols: human chorionic gonadotropin trends and patient outcomes. American journal of obstetrics and gynecology. 2016;215(5):590. e1-. e5.

32. Alyasin A, Badenoosh B, Agha-Hoseini M, Khademi F. Comparison of the success rate of ectopic pregnancy treatment with single dose and multiple dose methotrexate in Shariati Hospital 20032004. Tehran University Medical Journal. 2005;63(7):566-73.

33. Alexander JM, Rouse DJ, Varner E, Austin JM. Treatment of the Small Unruptured Ectopic Pregnancy: A Cost Analysis of Methotrexate Versus Laparoscopy. Obstetrical \& gynecological survey. 1997;52(2):109-10. 
34. Morlock RJ, Lafata JE, Eisenstein D. Cost-effectiveness of single-dose methotrexate compared with laparoscopic treatment of ectopic pregnancy. Obstetrics \& Gynecology. 2000;95(3):407-12.

35. Mol BW, Hajenius PJ, Engelsbel S, Ankum WM, Hemrika DJ, Van der Veen F, et al. Treatment of tubal pregnancy in the Netherlands: an economic comparison of systemic methotrexate administration and laparoscopic salpingostomy. American journal of obstetrics and gynecology. 1999;181(4):94551.

36. Mol F, Mol B, Ankum W, Van der Veen F, Hajenius P. Current evidence on surgery, systemic methotrexate and expectant management in the treatment of tubal ectopic pregnancy: a systematic review and meta-analysis. Human reproduction update. 2008;14(4):309-19.

37. Curran JW. Economic consequences of pelvic inflammatory disease in the United States. American journal of obstetrics and gynecology. 1980;138(7):848-51.

\section{Tables}

Table1. Demographic and background characteristics of the patients with ectopic pregnancy according to three treatment strategies

\begin{tabular}{|c|c|c|c|}
\hline Studied variables & Single-dose Methotrexate & Double-dose Methotrexate & surgery \\
\hline Number of patients & 116 & 68 & 70 \\
\hline Mean age of the patients & $29.9(5.75)$ & $31(5.2)$ & $30(4.81)$ \\
\hline Insurance coverage & $96.5 \%$ & $97 \%$ & $99 \%$ \\
\hline Number of parity & 2.15 & 2.56 & 2.64 \\
\hline Mean size of mass $\left(\mathrm{mm}^{3}\right)$ & 14.4 & 11.2 & 22.5 \\
\hline Mean age of gestation (day) & 50 & 49 & 51 \\
\hline History of pelvic surgery & $42 \%$ & $54 \%$ & $58 \%$ \\
\hline History of infertility & $20 \%$ & $16 \%$ & $14 \%$ \\
\hline History of ectopic pregnancy & $28 \%$ & $46 \%$ & $40 \%$ \\
\hline History of ectopic abortion & $9 \%$ & $9 \%$ & $13 \%$ \\
\hline
\end{tabular}

Table2. The Cost Components of treatment strategies 


\begin{tabular}{|c|c|c|c|c|}
\hline Costs & Costs items & $\begin{array}{c}\text { Single-dose Methotrexate } \\
\text { (PPP\$) }\end{array}$ & $\begin{array}{l}\text { Double-dose Methotrexate } \\
\text { (PPP\$) }\end{array}$ & $\begin{array}{l}\text { surgery } \\
\text { (PPP\$) }\end{array}$ \\
\hline \multirow[t]{6}{*}{ Direct medical costs } & Visit & 97 & 119 & 49 \\
\hline & Patient hospitalization & 1030 & 1226 & 580 \\
\hline & Laboratory tests & 267 & 263 & 111 \\
\hline & Radiography & 93 & 132 & 107 \\
\hline & Medication & 28 & 41 & 46 \\
\hline & Surgical services & 191 & 228 & 1430 \\
\hline $\begin{array}{l}\text { Direct non-medical } \\
\text { costs }\end{array}$ & $\begin{array}{c}\text { Transportation, accommodation and } \\
\text { meals }\end{array}$ & 431 & 684 & 229 \\
\hline \multirow[t]{2}{*}{ indirect costs } & Lost earnings & 538 & 669 & 607 \\
\hline & Total & 2675 & 3362 & 3159 \\
\hline
\end{tabular}

Table3: Comparing the cost-effectiveness of three methods of treating ectopic pregnancy by decision tree model

\begin{tabular}{|c|c|c|c|c|c|c|}
\hline & Strategy & $\begin{array}{l}\text { cost } \\
\text { (PPP\$) }\end{array}$ & effectiveness & Incremental Cost & Incremental effectiveness & $\begin{array}{l}\text { ICER } \\
\text { (Incremental } \\
\text { cost per extra } \\
\text { success) PPP\$ }\end{array}$ \\
\hline \multirow[t]{3}{*}{$\begin{array}{l}\text { Cost- Effectiveness } \\
\text { analysis (CEA) }\end{array}$} & $\begin{array}{l}\text { single-dose } \\
\text { methotrexate }\end{array}$ & 1984 & 0.721 & 0 & 0 & 0 \\
\hline & surgery & 2850 & 0.87 & 866 & 0.149 & 5812 \\
\hline & $\begin{array}{l}\text { double-dose } \\
\text { methotrexate }\end{array}$ & 3199 & 0.71 & 349 & -0.16 & abs. dominated \\
\hline
\end{tabular}

${ }^{*}$ ICER $=$ Incremental cost effectiveness ratio

Table 4: Comparing the cost-utility of the three methods of treating ectopic pregnancy by decision tree model 


\begin{tabular}{|c|c|c|c|c|c|c|}
\hline & Strategy & $\begin{array}{l}\text { cost } \\
\text { (PPP\$) }\end{array}$ & utility & Incremental Cost & Incremental utility & $\begin{array}{l}\text { ICUR (Incremental cost per QALY } \\
\text { Gained) PPP\$ }\end{array}$ \\
\hline \multirow{3}{*}{$\begin{array}{l}\text { Cost-Utility } \\
\text { analysis }\end{array}$} & surgery & 378 & 0.81 & 0 & 0 & c \\
\hline & $\begin{array}{l}\text { single-dose } \\
\text { methotrexate }\end{array}$ & 1968 & 0.827 & 1590 & 0.017 & 92191 \\
\hline & $\begin{array}{l}\text { double-dose } \\
\text { methotrexate }\end{array}$ & 3162 & 0.81 & 1194 & -0.017 & abs. dominated \\
\hline
\end{tabular}

${ }^{*}$ ICUR $=$ Incremental cost utility ratio

Figures 


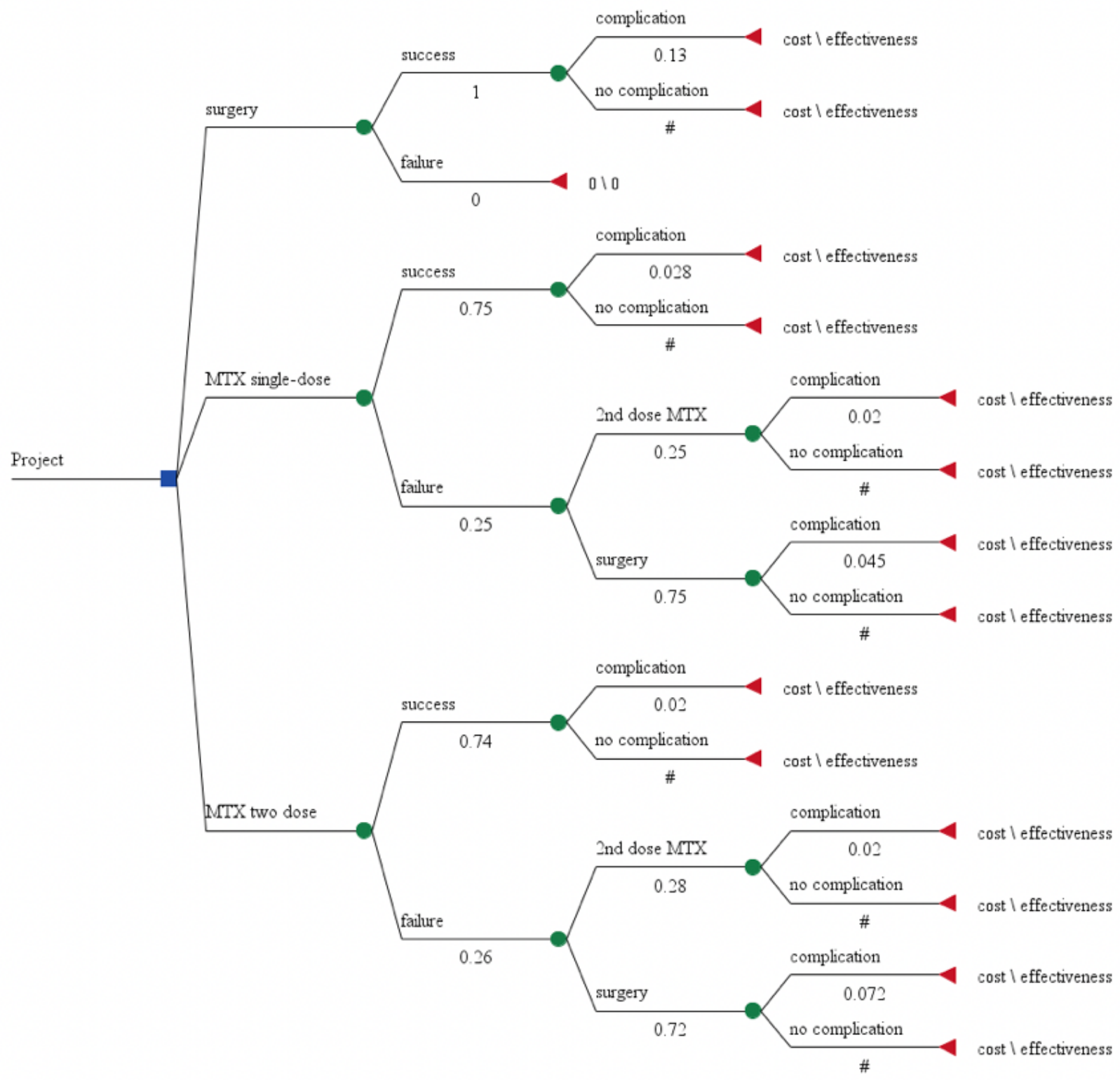

Figure 1

Decision tree model for different methods of treating ectopic pregnancy 
Cost-Effectiveness Analysis

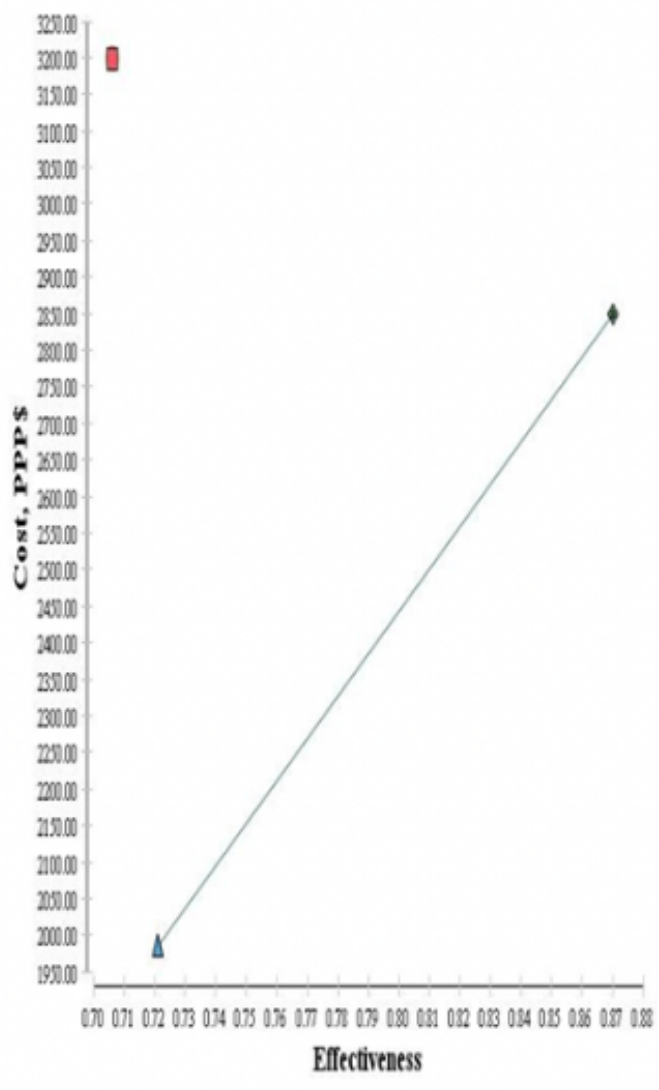

2-A
Cost-Effectiveness Analysis
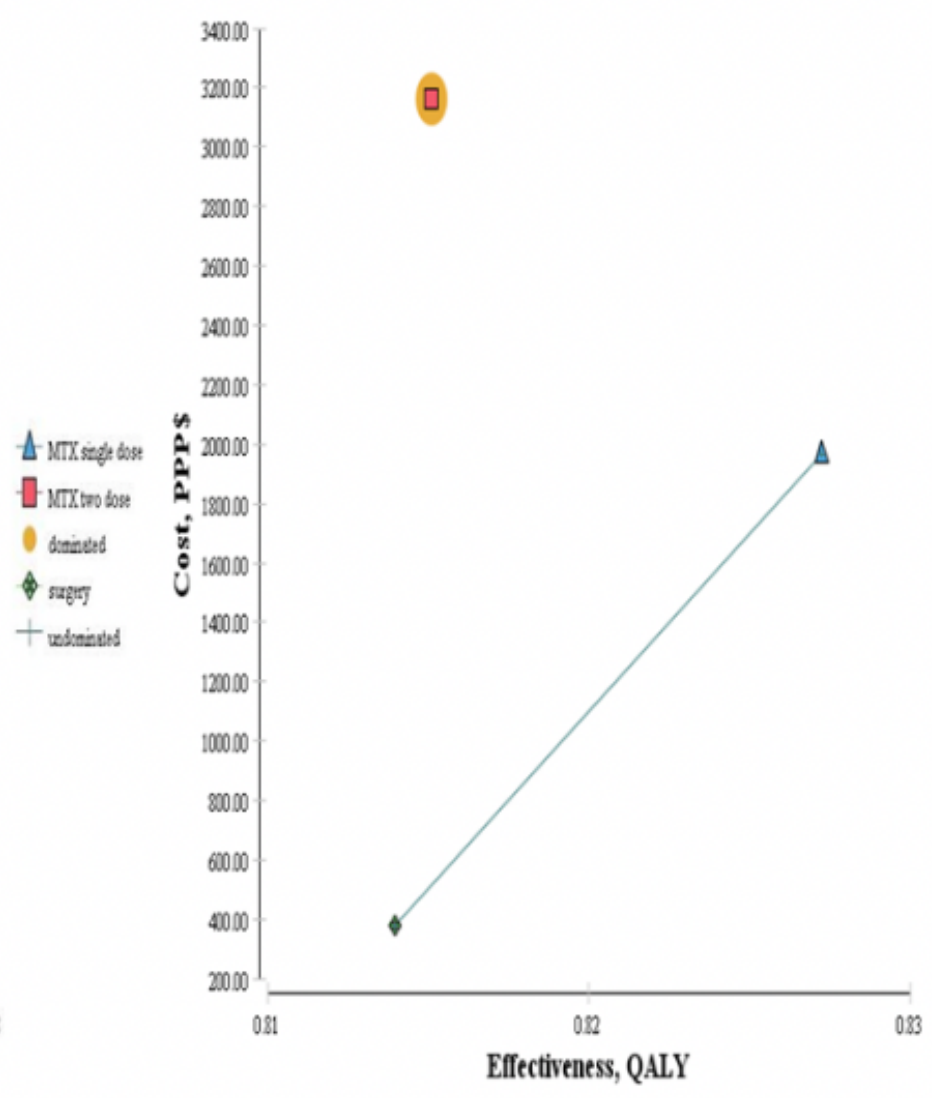

2-B

Figure 2

Cost-effectiveness (2-A) and cost-utility (2-B) analyses for ectopic pregnancy under drug therapy and surgical procedures 


\section{Tornado Analysis (ICER)}

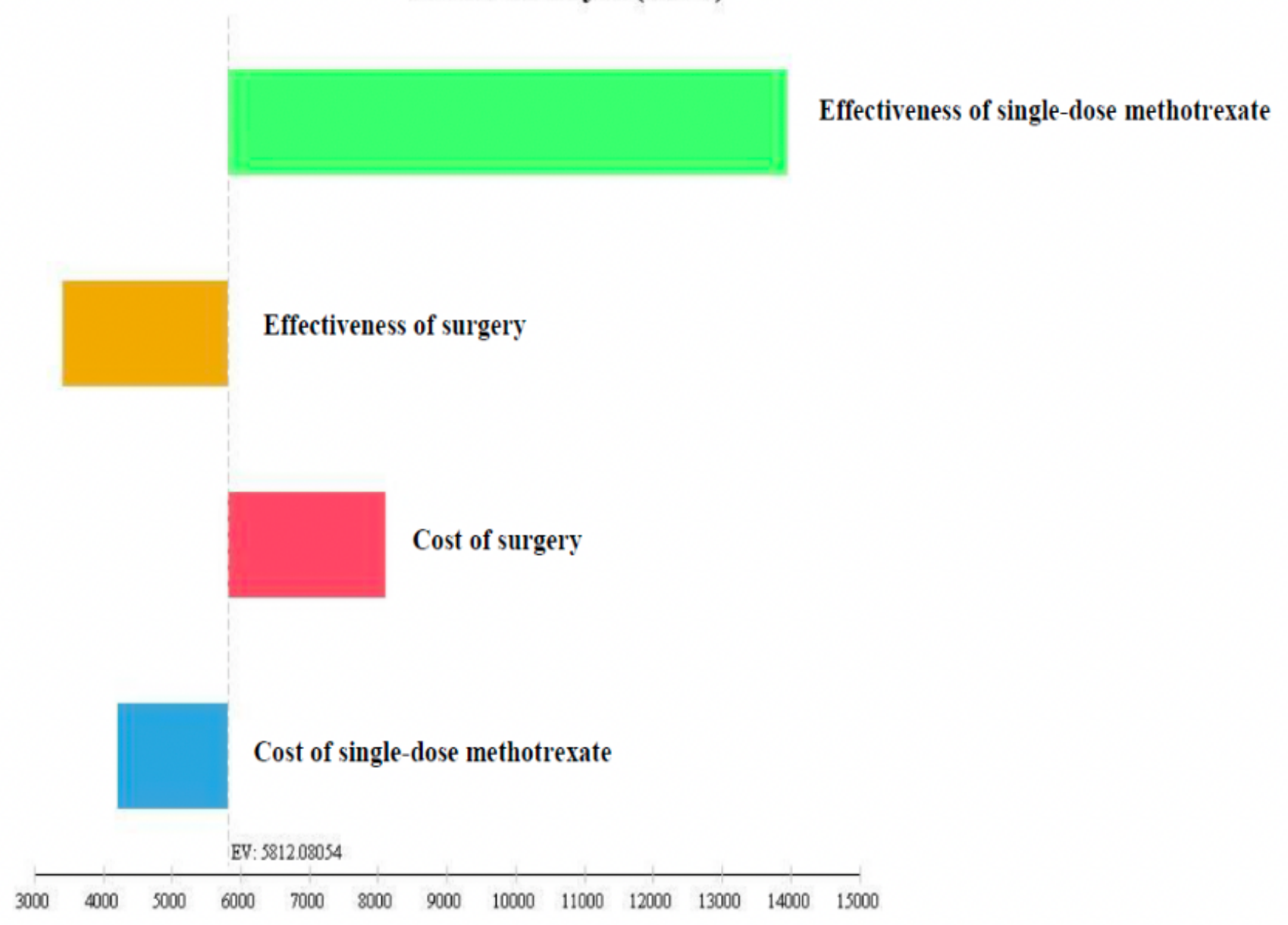

\section{Figure 3}

Tornado diagram of cost-effectiveness for patients with ectopic pregnancy under drug therapy and surgical procedures 


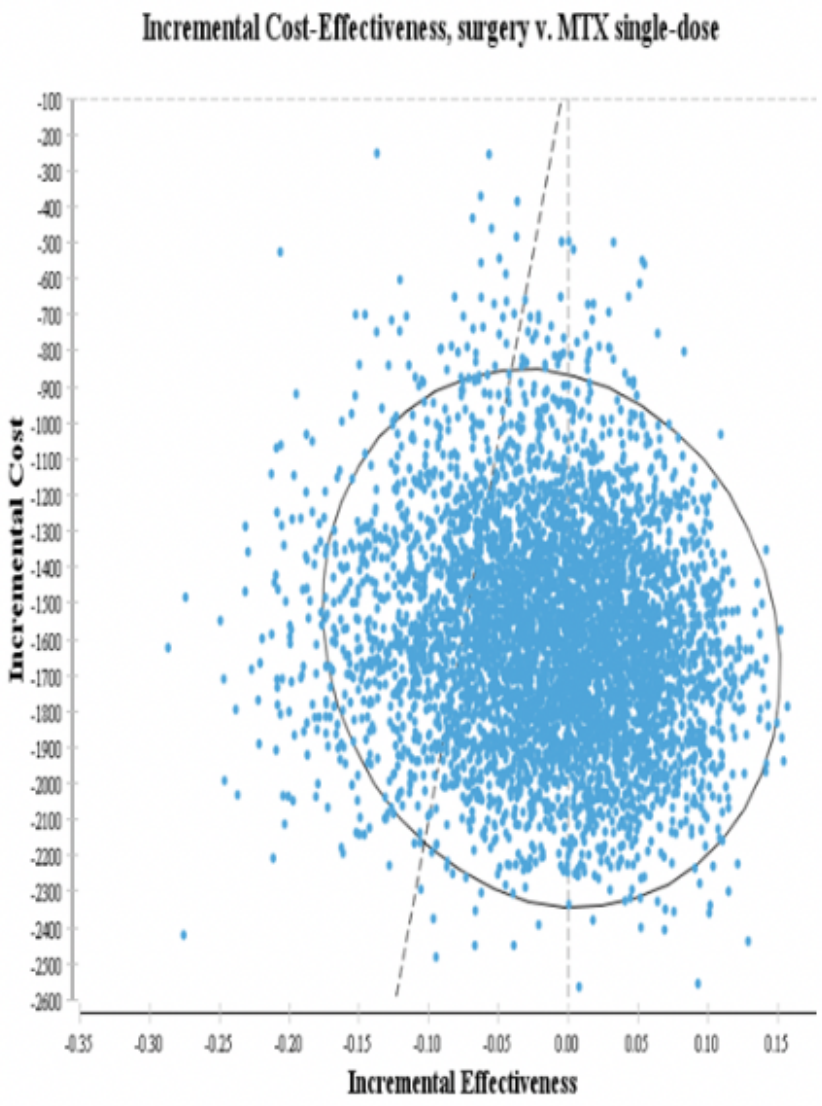

4-A
Incremental Cost-Efrectiveness, suggery v. MTX two dose

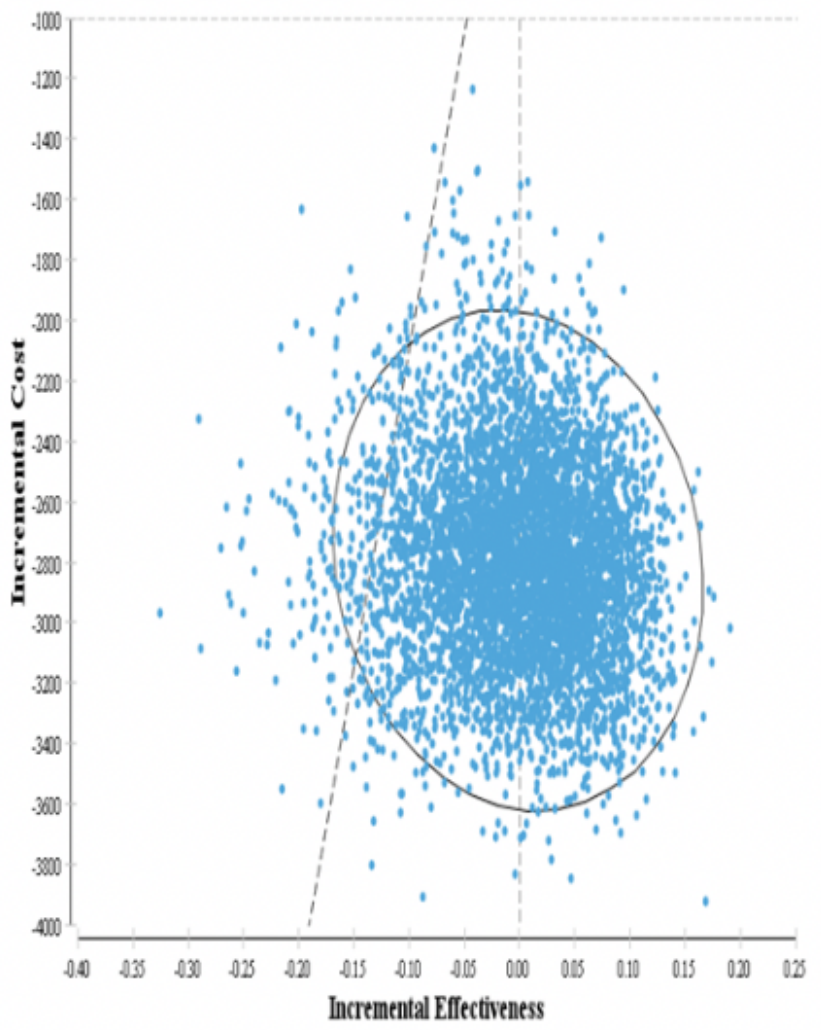

4-B

\section{Figure 4}

Incremental Cost-Effectiveness of surgery Compared with single- and double-dose methotrexate (4-A and 4-B) 


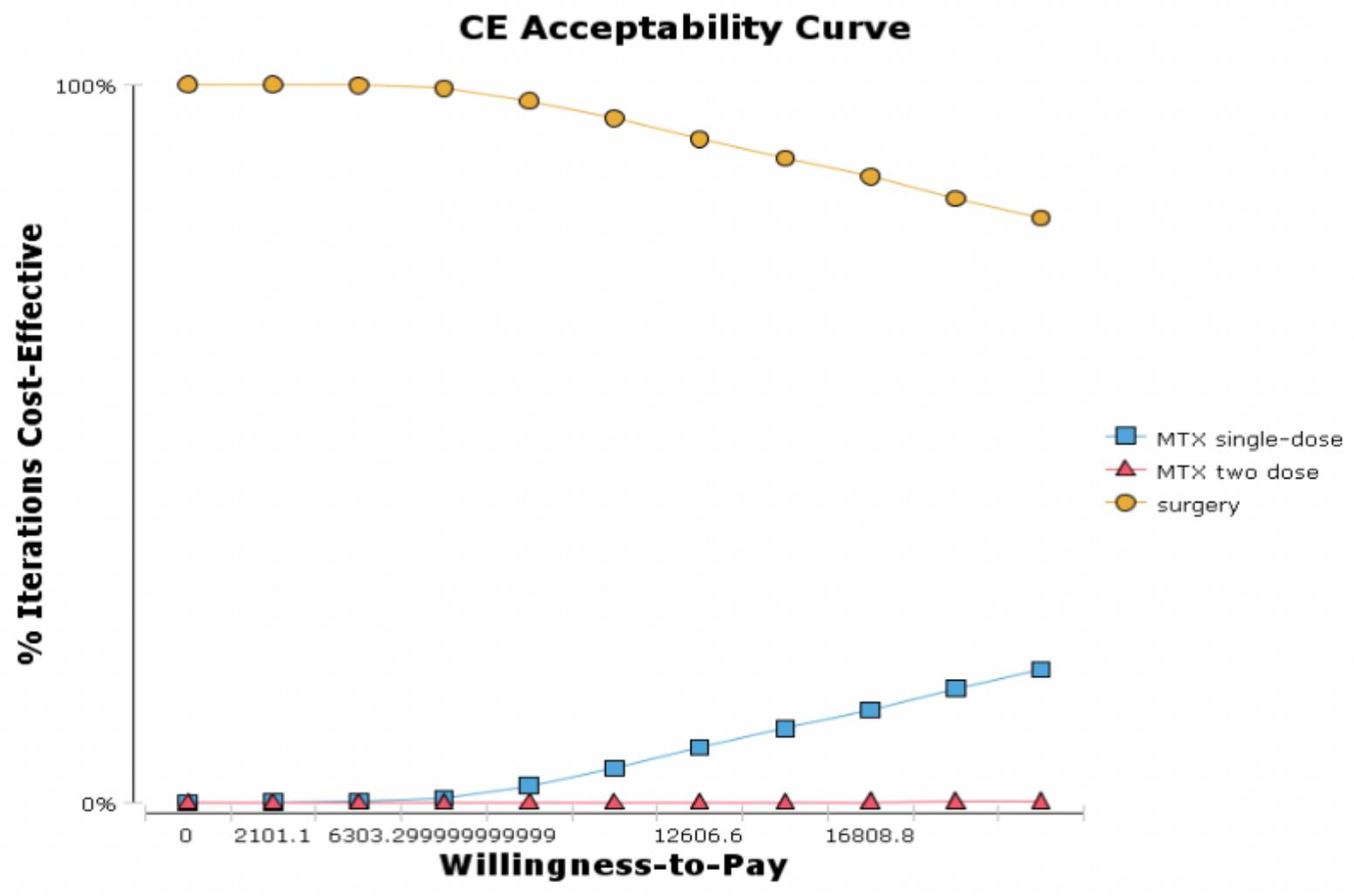

Figure 5

Cost-Effectiveness Acceptability Curve through Monte Carlo Simulation for patients with ectopic pregnancy under drug therapy 